\title{
Demand Response Shifting Management Applied to Distributed Generation and Pumping
}

\author{
Diogo Boldt, Pedro Faria and Zita Vale \\ dbpds@isep.ipp.pt,pnfar@isep.ipp.pt,zav@isep.ipp.pt \\ GECAD - Research Group on Intelligent Engineering and Computing for Advanced Innovation and \\ Development - Polytechnic of Porto (IPP) \\ R. Dr. António Bernardino de Almeida, 431, 4200-072 Porto, Portugal
}

\begin{abstract}
Recent energy policies in countries around the world, including in Europe, point to the need to integrate growing amounts of distributed generation in electric power systems. This situation led to several changes in the operation and planning of power systems. This paper presents a methodology focusing on demand response programs, distributed generation and pumping, which is aimed to be used by a Virtual Power Player, who is able to manage the available resources minimizing the operation costs. The influence of demand response shifting management, in which was possible to shift load from a critical period to other more benefic, was also taken into account. In this paper it was used Artificial Intelligence, Artificial Neural Networks (ANN), to predict the power the VPP would have to pump to reservoirs to fulfill the reservoir operator demands along the day. The case study includes 2223 consumers and 47 distributed generators units. The implemented scenario corresponds to a real day in Portuguese power system, $9^{\text {th }}$ March 2014.
\end{abstract}

\section{INTRODUCTION}

The smart grids (SG) are a field of study that are becoming more important and interesting, once it are associated to the development of the electric grid, in terms of network trustworthiness and in energy quality, which improves, significantly, its efficiency. The SG are proposed to evolve the grid reliability, once it are capable of controlling and managing the power generation and consumption of all the constituents. As it is described in [1], a SG pretends to introduce Distributed Generation (DG) in the electric grid by maximizing the penetration of renewable energy, but also Demand Response (DR) programs are being considered.

An important issue regarding the increase in the distributed generation units integration and, namely, wind power generation is the wind curtailment. Wind Curtailment is applied to wind power and it corresponds to the generation which isn't used, avoiding, this way, the possibility to achieve the maximum generation capacity of wind farms. If the available power is high and the wind farm operators opt to curtail the wind, it will result in a lower production, this procedure leads to an inefficient and negative impact in the use of the wind energy.

The present work was done and funded in the scope of the following projects: EUREKA - ITEA2 Project SEAS with project number 12004; H2020 DREAM-GO Project (Marie Sklodowska-Curie grant agreement No 641794); and UID/EEA/00760/2013 funded by FEDER Funds through COMPETE program and by National Funds through FCT.
A better comprehension of wind farms operators is essential to enhance the wind penetration of each country. The wind penetration concerns the fraction of the energy generated by the total production capacity available. The leader country in wind penetration, in Europe, is currently Denmark with $27 \%$. It is followed by Portugal and Spain, respectively with 17 and $16 \%$ [2]. The Wind Power installed in all Europe by the end of 2014 was 133,968 MW, while in 2013 and in 2012 was, respectively, $121,474 \mathrm{MW}$ and 109,581 MW, being that Germany, Spain and United Kingdom, along these years, are the ones that present the highest capacity installed [2] - [4]. To point out that the situation in Portugal, with the increase of renewable energy capacity, has been coming to meet the policies settled by the European Union.

This paper proposes a methodology focusing new and improved procedures to solve the wind curtailment problem, initially developed in [5]. According to the work that was previously developed it is important to point out that the main contributions of this paper mainly refer to the management of water storage in reservoirs, considering the pumping process and load shifting. Whenever generation is higher than expected, the Virtual Power Player (VPP) will be able to use it in pumping or initiate a Real Time Pricing (RTP) program, instead of, simply, curtail the wind. This measure can guarantee a better optimization of wind farms, while it also establishes a development in the use of clean energies. One particularity is the application of the shifting management of these last mentioned programs. Is given the opportunity for consumers to shift their loads between periods, since the main purpose is to incentive consumers to avoid the excessive consumption in critical periods [6]. As it is referred in [7] - [8], the process of pumping water to reservoirs is seen as an important measure to deal with the instability of power generation and demand. In periods where generation is higher and if hydroelectric power plants have significant capacity for pumping, then it is important to operate in this area, once it can be possible to pump water to reservoirs for further use. This measure besides seizing the excess generation it can mitigate future critical periods of demand, in the power system.

The method that was approached for further analysis it was the Artificial Neural Networks. What are Artificial Neural Networks? The Artificial Neural Networks (ANN) are composed by algorithms inspired in biological neural networks. It is one of several methods that consists in artificial intelligence, it was conceived to simulate the neuronal structure of a mammalian cerebral cortex, in a very small scale. The ANN has been in the field of study for a long period and one of the 
first neurocomputers, Dr. Robert Hecht-Nielsen, defined a neural network as: “.... computing system made up of a number of simple, highly interconnected processing elements, which process information by their dynamic state response to external inputs..." [9]. The ANN is used to determine the amount of water to be pumped to the reservoir; this method will be discussed in further detail in section II. For more information to supplement the knowledge about ANN is crucial to analyze the references given from [10] to [12].

After this introduction, Section II is divided into two subsections, A (Optimization) and B (Artificial Neural Networks), presenting the proposed methodology. In section III is presented the mathematical formulation applied to the problem, while in section IV it is described the case study. Section V presents the results obtained, while in VI are shown the conclusions of the paper.

\section{PROPOSED METHODOLOGY}

The methodology proposed is based in an optimization problem, using TOMLAB as a tool for results generation [13].

This paper proposes to study the maximization of generation resources referred as prioritary (non-storable), as the case of wind power, in a methodology developed to be used by a VPP (Virtual Power Player), an entity that is able to aggregate several types of distributed energy resources, such as DG, storage, DR and, most recently, electric vehicles [14]. It should be noted that this kind of units (prioritary) has production costs associated and it must be dispatched when the generation process occurs or else it is wasted, if not used.

\section{A. Scheduling Method}

To develop the optimization problem, it was taken into account some resources like distributed generation (DG) prioritary (non-storable) and other (storable), suppliers, reservoirs, pumping process. In addition to these, it were also considered programs that could be applied to consumers, as the case of Demand Response (DR) and Real-Time Pricing (RTP) programs. Figure 1 outlines the proposed and the developed methodology.

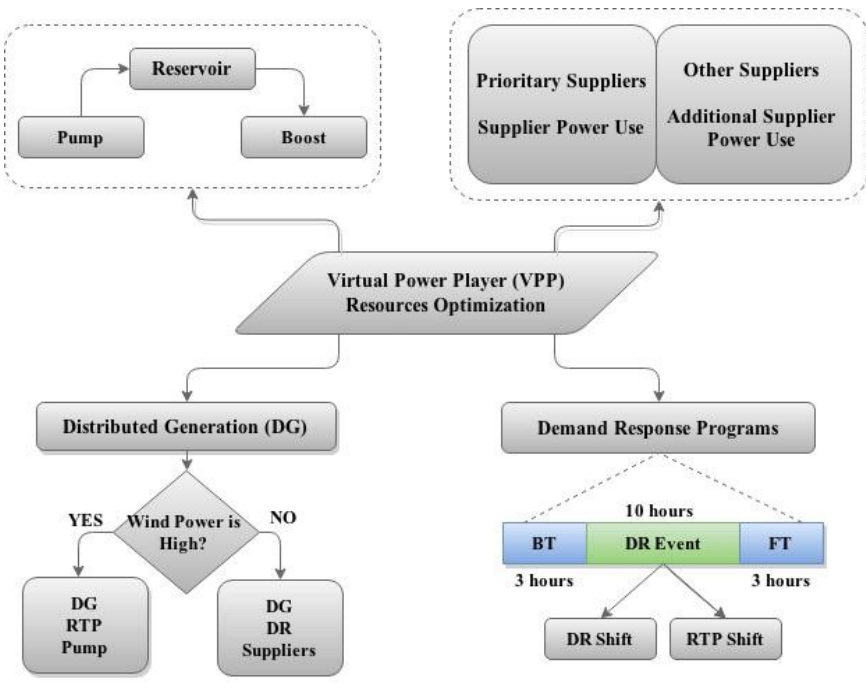

Figure 1 - Proposed Methodology diagram.
As said before, the generation would rely on the DG, which is divided into prioritary, non-storable, (Solar, Small Hydro, Wind and CHP) and other, storable, (CHP, Biomass, MSW) and on external suppliers, in case the available DG not be enough to meet the electric network requirement. For the reservoir, it was considered an initial state, for the primordial period, and it was expected that the amount stored was enough to fulfill the periods where the reservoir operator would opt to boost (turbine) water. To help in this case it was considered a pumping process, in which was given the possibility to store water into the reservoir, whenever the VPP feels more appropriate.

With the programs applied to consumers, DR and RTP, it is expected that they have the capacity to reduce, shift, or increase their loads, in periods where they find more attractive, in a financial way. With this method, it is possible to have a better network management, avoiding the excess of expensive generation. With DR it is expected that the consumers can reduce their loads, in periods where the generation is lower, in this case, the consumption also decreases, relieving load from the power system, during critical periods. Similar to the program presented before, DR shifting also intends to proceed to a better power system management in precarious periods, instead of reducing unnecessary load, it is shifted from critical periods to other day phases where the DG can easily supply the demand needs.

\section{B. Neural Network approach}

To conclude this paper, it was finally used Artificial Neural Networks (ANN) to predict the periods in which the VPP would opt to pump water to the reservoir. It is also expected that the ANN would predict the exact power to use in the pumping process that was necessary to fulfil the reservoir operator requirements. To consider scenarios for the ANN application it were assigned four different parameters: the alphas (alpha wind, alpha DG and alpha DR), as presented in chapter III, and the periods where the reservoir operator would opt to pump. These alphas represent percentage values that limit the quantity used of each resource by the VPP. With the deliberated variation it is expected that the VPP would dispatch the resources available according to the limitation that was assumed for each alpha. It were considered forty scenarios for the boost variation and three different values to each alpha, giving a total of 1080 scenarios. It is important to point out that in the optimization were considered 96 periods, while in the ANN were only considered 24 periods of the day. For this it were grouped 4 periods of 15 minutes and then it was calculated the average value, equalling it to one hour. For this specific situation it was expected that in the morning periods, 1 to 10 , the reservoir operator would not opt to pump water, it was assumed that the VPP knew that it was only possible to pump from period 11 to 24 . This procedure was stipulated to decrease the number of inputs the ANN would receive and then learn, the purpose was to achieve more interesting and better results, according to the inputs applied to the ANN. To conclude the simulation was used with Matlab Toolbox, nntool, and is presented a brief diagram in figure 2, presenting the parameters used. For more information is important to analyse [15]. 


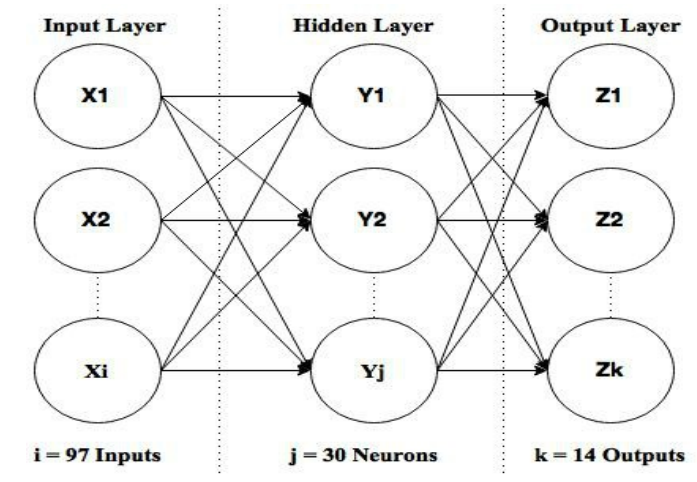

Figure 2 - Artificial Neural Network methodology diagram.

\section{MATHEMATICAL FORMULATION}

This section presents the mathematical formulation in order to minimize the VPP optimization problem costs. The objective function is presented in (1) and it considers DG, suppliers, DR, RTP, pump and consumption, among the 96 periods. The DG was divided into generation that can be storable (e.g.: wind, solar, small hydro), in prioritary DG, while the resources that are not storable (e.g.: Combined Heat and Power - CHP, Biomass, Municipal Solid Waste (MSW), in other DG. It is important to consider that all these equations are being applied to each scenario period, $t$. A detailed information about the case study will be presented in section $\mathrm{V}$.

$$
\begin{aligned}
& \text { Minimize } V P P_{O C}
\end{aligned}
$$

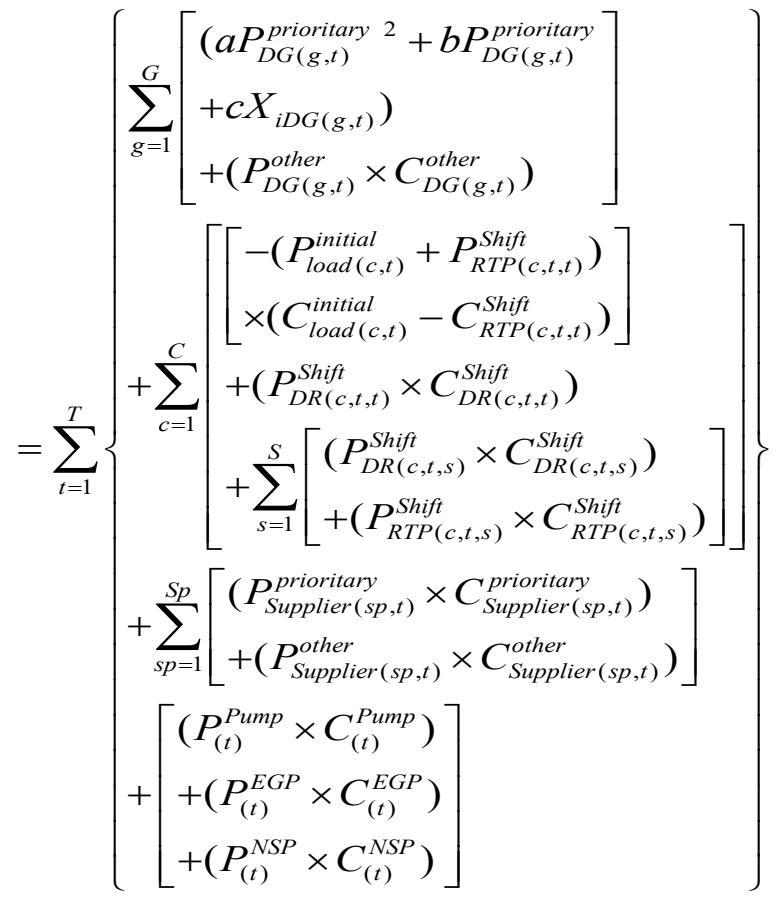

Setting the problem constraints correctly is one of the most important processes to achieve good results. The power balance constraint presented in (2) aims to equal the generation to consumption, in this way the VPP ensures a more efficient use of the available resources, satisfying the required demand.

$$
\begin{aligned}
& \left.\sum_{g=1}^{G}\left[P_{D G(g, t)}^{\text {prioritary }}+P_{D G(g, t)}^{\text {other }}\right)\right] \\
& +\sum_{s p=1}^{S p}\left[\left(P_{\text {Supplier }(s p, t)}^{\text {prioritary }}+P_{\text {Supplier }(s p, t)}^{\text {other }}\right]\right. \\
& =\sum_{c=1}^{C}\left[\begin{array}{c}
\left(P_{\text {load }(c, t)}^{\text {initial }}+P_{R T P(c, t, t)}^{\text {Shift }}-P_{D R(c, t, t)}^{\text {Shift }}\right. \\
\left.+\sum_{s=1}^{S}-\left(P_{D R(c, t, \mathrm{~s})}^{\text {Shift }}-P_{R T P(c, t, \mathrm{~s})}^{\text {Shift }}\right)\right)
\end{array}\right]+P_{(t)}^{\text {Pump }}
\end{aligned}
$$

The constraints modeled from (3) - (6) refer to the elasticity and the generation. The constraint in (3) presents the price elasticity of demand of each consumer. It is a restriction that responds with the changes of other variables, for example, if the demand is high, so will it be the energy price, or if the generation is high it is expected that the energy price decreases. The other constraints, from (4) - (6) are applied to DG, in which obligates the VPP to not exceed the maximum generation capacity. In (5), the equality is considered to maximize the use of prioritary DG resources, such as wind, solar or small hydro once they cannot be stored.

$$
\begin{aligned}
& \varepsilon=\frac{P_{\text {Demand }(c, t)}^{\text {increase }} \times P_{\text {Demand }(c, t)}^{\text {initial }}}{P_{\text {Demand }(c, t)}^{\text {forecast }} \times P_{\text {Demand }(c, t)}^{\text {decrease }}} \\
& P_{D G(g, t)}^{\text {prioritary }}+P_{D G(g, t)}^{\text {other }} \leq P_{D G(g, t)}^{\text {Max }} \\
& P_{D G(g, t)}^{\text {prioritary }}+P_{E G P(t)}^{\text {prioritary }}=P_{D G(g, t)}^{\text {prioritaryMax }} \\
& P_{D G(g, t)}^{\text {others }} \leq P_{D G(g, t)}^{\text {OthersMax }}
\end{aligned}
$$

The constraints (7) - (9) point to the demanded energy required to suppliers, which cannot be higher than the limits stipulated. As described in the equations, the prioritary, other and the sum of both suppliers are limited with a maximum value, in order to ensure that the active power used is between the considered ranges.

$$
\begin{aligned}
& P_{\text {Supplier }(s p, t)}^{\text {prioritary }}+P_{\text {Supplier }(s p, t)}^{\text {other }} \leq P_{\text {Supplier }(s p, t)}^{\text {Max }} \\
& P_{\text {Supplier }(s p, t)}^{\text {prioritary }} \leq P_{\text {Supplier }(s p, t)}^{\text {prioritaryMax }} \\
& P_{\text {Supplier }(s p, t)}^{\text {other }} \leq P_{\text {Supplier }(s p, t)}^{\text {otherMax }}
\end{aligned}
$$

The constraints from (10) - (15) refer to the pumping process applied in periods where the generation is higher than demand. The restriction (11) presents the possibility to pump water, knowing if the reservoir operator is boosting water, since it can only occur one action at a certain period. In (12), (13) is restrained the reservoir level, maintaining the relation between the reservoir capacity with the pumping and turbine process. While in (14) it is considered a balance equation to present the reservoir level, in a period $t$, taking into account the state level in the previous period ( $\mathrm{t}-1)$ and the pump or boost process that may occur. The last equation, (15), assumes an initial reservoir state, when period is $\mathrm{t}=0$. 
$P_{(t)}^{\text {reservoir }} \leq P_{(t)}^{\text {reservoirMax }}$

$P_{(\mathrm{t})}^{\text {Pump }} \leq P_{(t)}^{\text {PumpMax }}, P_{(t)}^{\text {Pump }}=0$ when $P_{(t)}^{\text {Boost }}>0$

$P_{(t)}^{\text {Pump }}+P_{(t-1)}^{\text {reservoir }} \leq P_{(t)}^{\text {reser }}$

$P_{(t)}^{\text {Boost }}-P_{(t-1)}^{\text {reservoir }} \leq P_{(t)}^{\text {reservoirMin }}$

$P_{(t)}^{\text {reservoir }}=P_{(t-1)}^{\text {reservoir }}+P_{(t)}^{\text {Pump }}-P_{(t)}^{\text {Boost }}$

$P_{(t=0)}^{\text {reservoir }} \leq P^{\text {reservoir_InitialState }}$

The constraints from (16) - (18) refer to Demand Response programs applied to the consumers. In constraint (16) is presented the maximum limit of DR that can be shifted from a period $t$ to a period $s$. In situations where the period t equals period $\mathrm{s}(\mathrm{t}=\mathrm{s})$ is considered reduction instead of the shifting. In (17), the total DR can be shifted from a period t to each one of the periods s. While in (18), it represents the maximum value of DR shifted for each period s, according to each consumer type c.

$$
\begin{aligned}
& P_{D R(c, t, s)}^{\text {Shift }} \leq P_{D R(c, t, s)}^{\text {ShiftMax } t \rightarrow s} \\
& \sum_{\substack{t-B S \leq s \\
s \leq t+F S}}^{s \leq t+F S} P_{D R(t, s, \mathrm{c})}^{\text {Shift }} \leq P_{D R(t, c)}^{\text {ShiftMax } t} \\
& \sum_{t-B S \leq s}^{\text {Shift }} P_{D R(s, t, c)}^{\text {SR }} \leq P_{D R(t, c)}^{\text {ShiftMax } s}
\end{aligned}
$$

In (19) - (21), the constraints concern the RTP program, which is also related to the consumers, and their application field is similar to the DR equations, (16) to (18), presented previously.

$$
\begin{aligned}
& P_{R T P(c, t, s)}^{\text {Shift }} \leq P_{R T P(c, t, s)}^{\text {ShiftMax } t \rightarrow s} \\
& \sum_{t-B S \leq s}^{s \leq t+F S} P_{R T P(t, s, \mathrm{c})}^{\text {Shift }} \leq P_{R T P(t, c)}^{\text {ShiftMax } t} \\
& \sum_{t-B S \leq s}^{s \leq t+F S} P_{R T P(s, t, c)}^{\text {Shift }} \leq P_{R T P(t, c)}^{\text {ShiftMax } s}
\end{aligned}
$$

The equations (22) - (24) represent maximum limits, in percentage. This percentage value represents the maximum contribution that each resource can have in the resources scheduling according to the VPP goals.

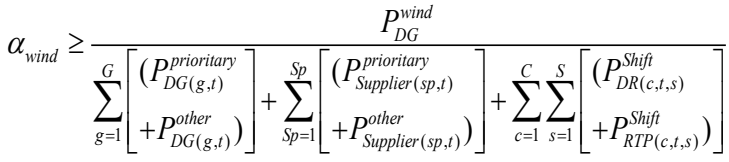

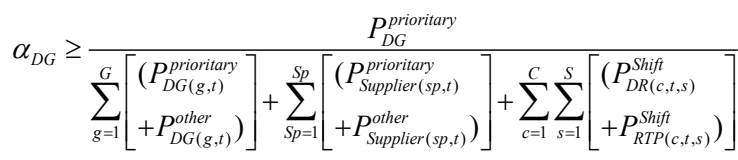

$$
\begin{aligned}
& \alpha_{D R} \geq \frac{P_{D R}}{\sum_{g=1}^{G}\left[\begin{array}{l}
\left(P_{D G(g, t)}^{\text {prioritary }}\right. \\
\left.+P_{D G(g, t)}^{\text {other }}\right)
\end{array}\right]+\sum_{S p=1}^{S p}\left[\begin{array}{l}
\left(P_{\text {Supplier }(\text { sp }, t)}^{\text {prion }}\right. \\
\left.+P_{\text {Supplier }(s, t)}^{\text {other }}\right)
\end{array}\right)+\sum_{c=1}^{C} \sum_{s=1}^{S}\left[\begin{array}{l}
\left(P_{D R(c, t, s)}^{\text {Shift }}\right. \\
\left.+P_{R T P(c, t, s)}^{\text {Shift }}\right)
\end{array}\right]}
\end{aligned}
$$

\section{CASE STUDY}

The current section will present a detailed explanation about the case study focusing the considered proposed methodology. The work was based on a real Portuguese distribution network where only the Feeder 5 was used to simulate the scenario, 9th March 2014. The chosen scenario represents a great day in the Portuguese electrical system where the generation of renewable energy was higher than the demand. The Feeder 5 is composed by 2223 consumers, divided into five types of consumers and 47 DG. The tables presented below intend to schematize the network constitution with higher detail. This scenario presents the adequate conditions in which is possible to apply the proposed methodology. It is important to emphasize that the demand in this network was about $5384 \mathrm{~kW}$, while the power of the available DG is $3359 \mathrm{~kW}$.

The tables 1 and 2, will schematize the parameters that were

\begin{tabular}{|c|c|c|c|c|c|}
\hline $\begin{array}{c}\text { Consumers } \\
\text { Characteristics }\end{array}$ & Domestic & $\begin{array}{c}\text { Small } \\
\text { Commerce }\end{array}$ & $\begin{array}{c}\text { Medium } \\
\text { Commerce }\end{array}$ & $\begin{array}{c}\text { Large } \\
\text { Commerce }\end{array}$ & Industrial \\
\hline $\begin{array}{l}\text { Number of } \\
\text { Consumers }\end{array}$ & 1107 & 1083 & 8 & 9 & 16 \\
\hline $\begin{array}{l}\text { Peak Consumption } \\
(\mathrm{kW})\end{array}$ & 780 & 777 & 851 & 746 & 2230 \\
\hline Elasticity & 0,27 & 0,33 & 0,37 & 0,41 & 0,53 \\
\hline $\begin{array}{l}\text { Initial Price } \\
\text { (m.u./kWh) }\end{array}$ & 0,21 & 0,18 & 0,20 & 0,19 & 0,15 \\
\hline $\begin{array}{l}\text { Maximum Power } \\
\text { Increase }(\mathrm{kW}) \\
(\text { Pincrease Mix })\end{array}$ & 2340,4 & 6217,9 & 6810,9 & 5971,2 & 17844,9 \\
\hline $\begin{array}{c}\text { Maximum Price } \\
\text { Variation } \\
\left(\text { Cdecrease Max }^{2}\right)\end{array}$ & 1,05 & 0,9 & 1 & 0,95 & 0,75 \\
\hline $\begin{array}{l}\text { Maximum DR } \\
\text { Reduction }(\mathrm{kW})\end{array}$ & 0,513 & 1,871 & 161,39 & 102,46 & 63,65 \\
\hline $\begin{array}{c}\text { DR Costs } \\
\text { (m.u./kWh) }\end{array}$ & 0,2 & 0,16 & 0,19 & 0,18 & 0,14 \\
\hline
\end{tabular}
taken into account for the optimization development. The first table is applicable to the DG production, while the second it is referable to the consumers.

Table 1 - Producers Characteristics.

\begin{tabular}{|c|c|c|c|c|}
\cline { 2 - 5 } \multicolumn{1}{c|}{} & $\begin{array}{c}\text { Distributed } \\
\text { Generators }\end{array}$ & $\begin{array}{c}\text { Generation } \\
\text { cost } \\
\text { (m.u./kWh) }\end{array}$ & $\begin{array}{c}\text { Number of } \\
\text { generation units }\end{array}$ & $\begin{array}{c}\text { Installed } \\
\text { capacity } \\
\text { (kVA) }\end{array}$ \\
\cline { 2 - 5 } Prioritary & Supplier & $0,23-0,32$ & 10 & 30000 \\
\hline \multirow{5}{*}{ Other } & Photovoltaic & 0,15 & 18 & 610 \\
& Wind & 0,071 & 22 & 508 \\
& Small hydro & 0,042 & 2 & 7 \\
& CHP & 0,00106 & 0.9 & 1000 \\
& Biomass & 0,086 & 2 & 226 \\
& MSW & 0,056 & 1 & 8 \\
& CHP & 0,3 & 0.1 & 1000 \\
\hline
\end{tabular}

Table 2 - Consumers Characteristics.

Recalling what was previously presented in this paper, in several sections, in the context of the problem it were considered distinct resources like: distributed generation (DG), programs of Demand Response (DR) and Real-Time Pricing (RTP), External Suppliers and reservoirs.

The main objective is to apply a Virtual Power Player (VPP) in order to perform a resources schedule, considering the minimization of the operations costs. In section VI.A it is 
possible to verify the base scenario that was chosen for the development of this optimization problem.

The 24 hours of the day are divided in 96 periods of 15 minutes. The considered resources are available for the 96 periods. In situations where the production overlaps the consumption (DG>Load), the VPP may opt to initiate a pumping process to consume excess generation, storage water in the reservoir, or to encourage the consumers to increase their loads (RTP program), to prevent large energy losses.

Meanwhile, the DR and RTP programs in which was capable to apply the shifting management to the industrial consumers (see table 3), were deliberated for the last 60 periods of the day (36 to 96), explicitly, between 8:45 AM to 11:45 PM. The DR and RTP were only available for the period 49 to 83 (10 hours), it was also considered the backward (BT) and forward (FT) time horizons, for the situations immediately before and after the consumers programs. For the BT and FT it were measured 3 hours, which corresponds to the periods 36 to 48 and periods 84 to 96 , respectively. The BT is intended to inform, previously, the consumers of the possibility to participate in the programs presented before. To perform a better result analysis, it was created two different clusters for the industrial type of consumers. The first eight consumers will be considered for DR, while the last eight will be deliberated to a RTP program. Table 3 pretends to demonstrate the distinct programs that were applied to the different types of consumers.

Table 3 - Consumers Programs.

\begin{tabular}{|c|c|c|c|c|c|}
\cline { 2 - 6 } \multicolumn{1}{c|}{} & 1107 & 1083 & 8 & 9 & 16 \\
\cline { 2 - 6 } \multicolumn{1}{c|}{} & Domestic & $\begin{array}{c}\text { Small } \\
\text { Commerce }\end{array}$ & $\begin{array}{c}\text { Medium } \\
\text { Commerce }\end{array}$ & $\begin{array}{c}\text { Large } \\
\text { Commerce }\end{array}$ & Industrial \\
\hline$P_{D R(\mathrm{c}, \mathrm{t})}^{\text {Red }}$ & $\mathrm{X}$ & $\mathrm{X}$ & & & $\mathrm{X}$ \\
\hline$P_{D R(\mathrm{t}, \mathrm{s}, \mathrm{c})}^{\text {Shift }}$ & & & & & $\mathrm{X}$ \\
\hline$P_{R T P(\mathrm{t}, \mathrm{s}, \mathrm{c})}^{\text {Shif }}$ & & & & $\mathrm{X}$ & \\
\hline$P_{R T P(c, t)}^{\text {increase }}$ & & & $\mathrm{X}$ & \\
\hline
\end{tabular}

Withal, were applied also DR and RTP to the rest of the consumers, as described in table 3 . For the type of consumers Domestic and Small Commerce is given the possibility to reduce their loads, in situations where they find it can be economically profitable or in situations where the electric network faces critical periods. The last measure, according to the table 3, is based in RTP but it is only applicable to Medium and Large Commerce and it aims to increase the demand, in periods where the generation is high. The VPP to encourage these consumers to use this program, it decides to decrease the energy price, becoming more attractive for these consumers to increase their consumption. In [5] is presented a similar application of demand response shifting management.

\section{RESULTS}

This section is intended to present the results that were obtained with the present study case. It is important to refer that all the outcomes concerns several situations regarding the base scenario, an example scenario to focus on the details of the resources scheduling, and the sensitive analysis for several scenarios. All these are related to the real Portuguese scenario in 9th March 2014.

\section{A. Base Scenario}

In order to present a detailed description of the obtained results, it was selected an example scenario referred as base scenario. This scenario corresponds to a real day in the Portuguese Power Systems, 9th March 2014. It was important to use the exact values of the day in study, to approach the optimization to a real situation to achieve promising results. The figure 3, represents the 24 hours of that day, in 96 periods of 15 minutes, as seen previously. It is necessary to understand that this scenario is capable to feed the demand, in the morning period, using only the DG available, however, on the second half of the day, the demand surpasses the electric network generation capacity, in this way it cannot fulfil the necessity to provide the consumers requirement.

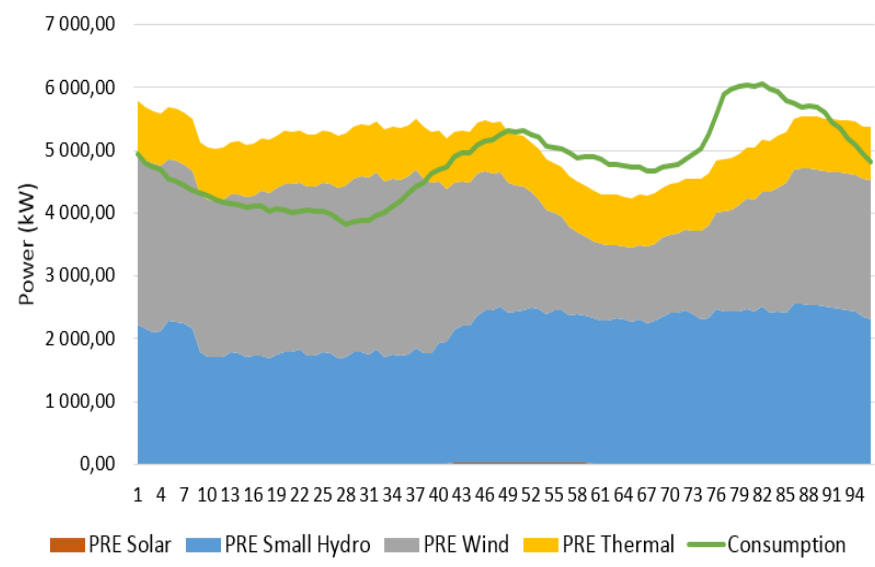

Figure 3 - Base Scenario, 9th March 2014 [16].

\section{B. Resources Scheduling}

This section presents the resources scheduling for the day in study. In figure 4 it is possible to analyze that all the production equals the consumption, fulfilling what was stipulated in the constraints, along the 96 periods. The VPP is with constant concern as regards the maximization of renewable resources, namely the prioritary ones (Solar, Small Hydro, Wind and Combined Heat and Power-CHP), in which the wind and small hydro represent the most used.

It is also interesting to analyze that the Medium and Large Commerce were also capable to increase their consumption in the more periods, when according to the base scenario, the DG is higher than the demand. 


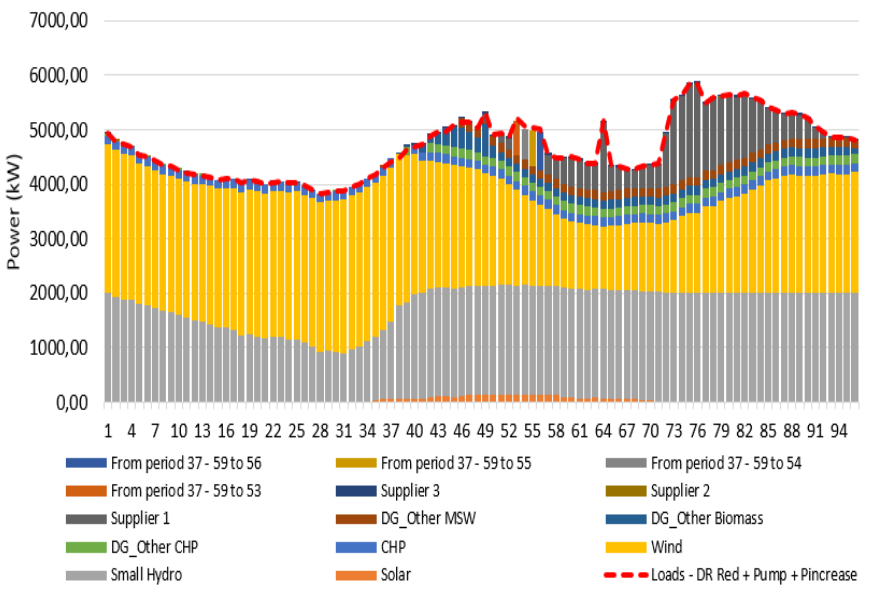

Figure 4 - Resources scheduling, along the 96 periods.

The figure 5 is similar to the figure that was previously presented, it represents the resources schedule from the VPP, but only for the last 46 periods of the day, where the demand was higher than generation. It is important to emphasize that, as described in the following figure, the loads in the second period of the day have decreased, essentially, due to the DR program, in which the consumers (Domestic and Small Commerce) decided to participate in. On the other hand, the periods where consumption surpasses the initial load, at least in two moments, is due to the pumping process initialized from the VPP, to fulfill the requirement from the reservoir operator, in which it may be imminent the use of water in reservoir to generate energy (boost). For a better comprehension is important to compare the moments in which the VPP opts to pump water to the reservoir, described in figure 6 .

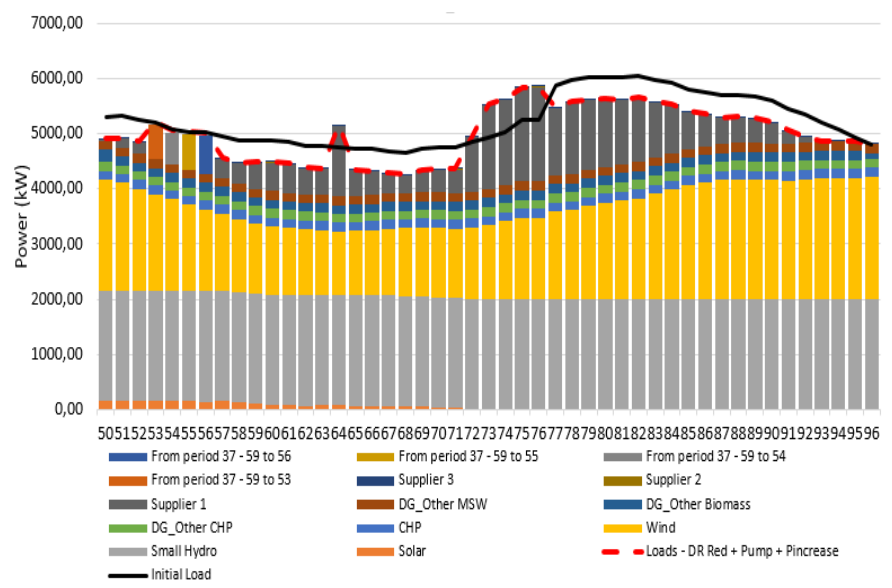

Figure 5 - Resources scheduling, last 46 periods.

For a period $t$, where the water from the reservoir is turbined, the VPP must ensure in previous intervals $(\mathrm{t}-1)$ that it already exists the amount required, in storage. So the visible peaks, in figure 5 that surpass the loads, is when the VPP opts to pump water to the reservoir, as seen before. In figure 6 it is shown the reservoir level, along the 96 periods. The variation is related with the amount used to turbine water (Boost) or the quantity of water that is pumped to the reservoir. It is important to enhance that, for this study case, it was considered for the primary period $(t=0)$ an initial reservoir state.

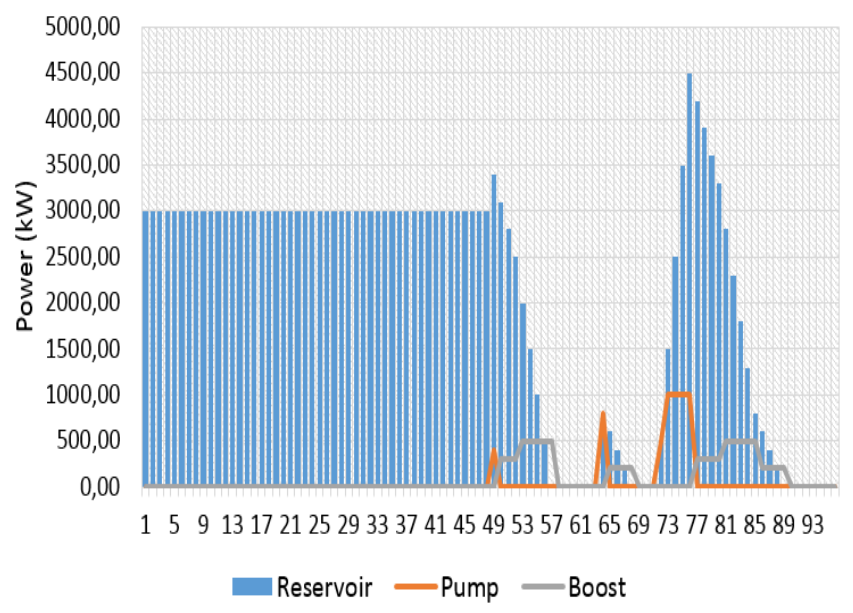

Figure 6 - Reservoir usage, along the 96 periods.

\section{Shifting Management - DR and RTP program}

The current subsection pretends to focus on the results obtained with the shifting management applied to the industrial consumers, while using the demand response program, as it is possible to analyze in figure 7.

The shifting management was effective, due to the change in the consumers behavior, as seen in case study (see Chapter IV), this measure is only available in the last 60 periods of the day. It is possible to analyze in the following figure that the industrial consumer intended to shift the consumption in period $37-41$ to the periods $49-52$.

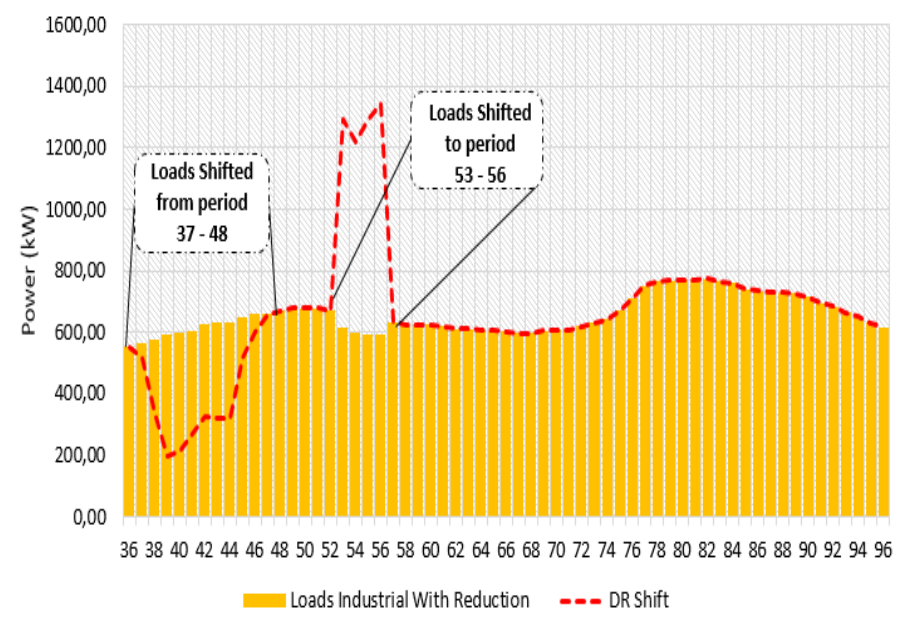

Figure 7 - Shifting Management applied to DR.

The following figure 8 is related with the RTP Shift and it can be possible to analyze that, the other industrial consumer also opted to shift their loads from the periods $37-48$ to the periods $53-56$, although in a very small scale, when compared to the figure 7. This consumer only opted to shift their loads, avoiding the possibility to also reduce, in critical periods. 


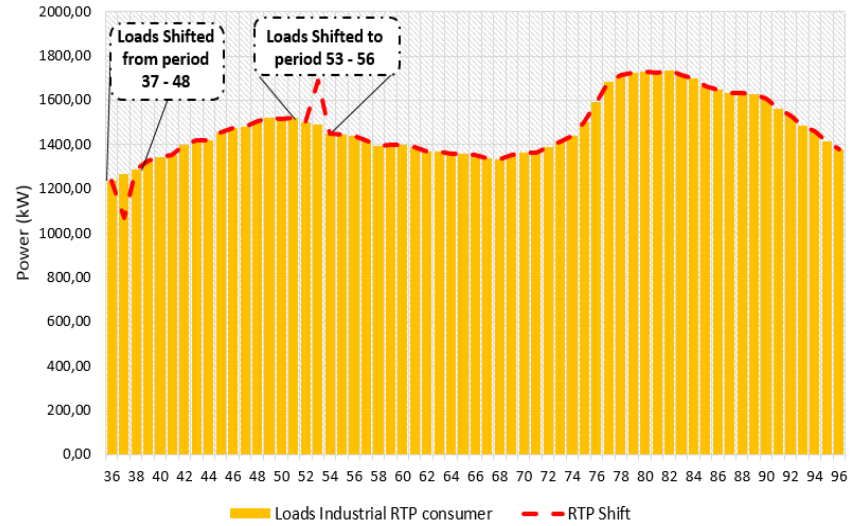

Figure 8 - Shifting Management applied to RTP.

\section{Artificial Neural Networks}

This subsection is intended to present the results obtained with the simulation of the ANN. It is expected that the results show the periods in which is more viable to pump to the reservoir, according to the scenario that the ANN is being tested. For this simulation it was considered the periods in which the reservoir operator would boost water, the variation between the considered alphas - Wind, DG and DR - but also the periods where the DG equals the electric network demand and the energy price variation of the suppliers. It is important to point out that all the parameters were assumed for the different values for each alpha scenario.

The figures 9.a) and 9.b) present the results obtained while running the ANN. It is expectable that the ANN with the given inputs can predict the amount of power used, when compared to the real values. In table 4 is schematized the scenarios that were taken into account to apply to the ANN as inputs. With these scenarios the amount of each resource would be different in each case, which was important for the ANN to receive diverse situations, as an input, for the learning process.

Table 4 - Scenarios Characterization.

\begin{tabular}{|c|c|c|c|c|}
\hline \multicolumn{5}{|c|}{ Scenarios considered } \\
\hline PBoost & $\alpha$ Wind & $\alpha$ DG & $\alpha$ DR & Total \\
\hline 40 & 3 & 3 & 3 & 1080 \\
\hline
\end{tabular}

The difference between the figures 9.a) and 9.b) is the period where the reservoir operator would opt to boost and the amount of power boosted, which is different in the several periods and scenarios, from the reservoir and the alphas that each scenario is subjected, as seen before. It is expected that the VPP operator would pump water to ensure that the amount needed is stored in the reservoir, according to the percentage used for each resource, which are limited by the three alphas. The figures presented are two examples of the vast results obtained from the ANN.

As was mentioned before, to achieve better results with a more realistic scenario, the ANN was trained only for the known periods in which the reservoir operator would opt to pump - period 11 to 24 .
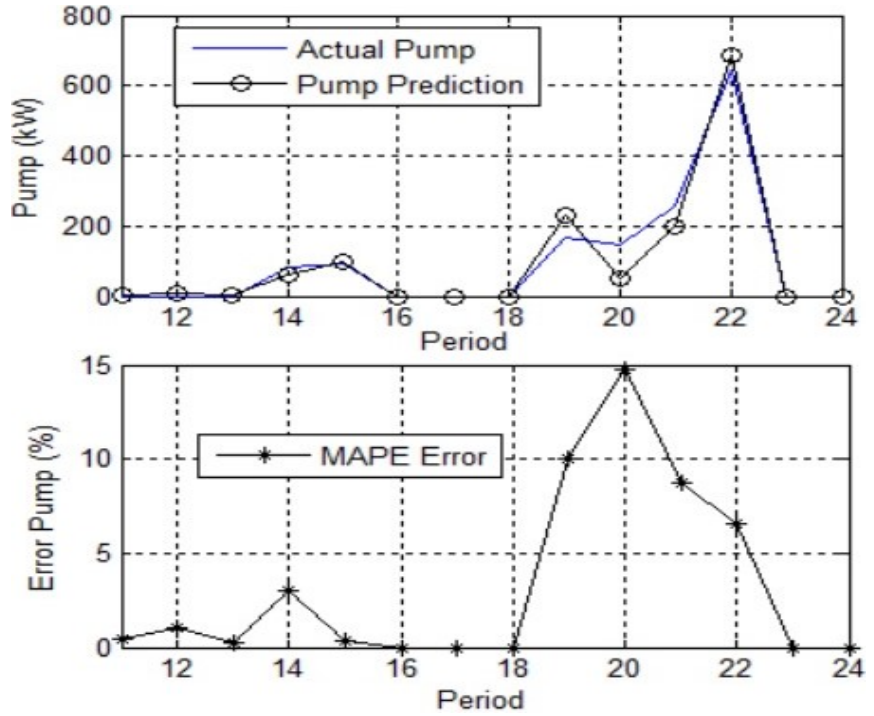

Figure 9.a) - ANN results for Cen651.
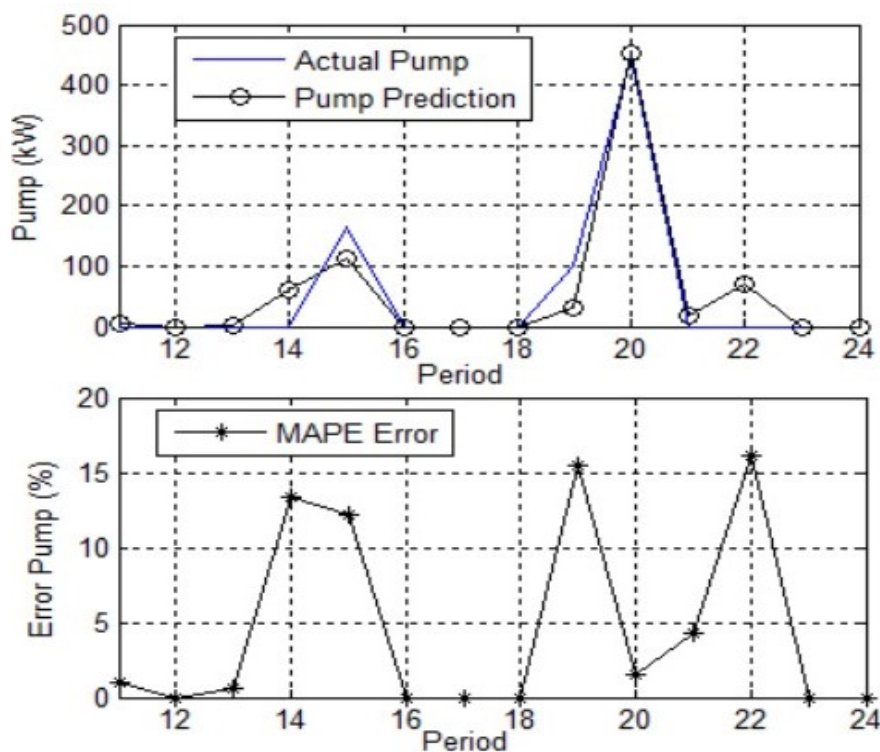

Figure 9.b) - ANN results for Cen1051.

To analyze the results that were obtained, when relating the actual values with the ones predicted, it is important to point out that is was used the MAPE (Mean Absolute Percentage Error). The MAPE is known for being a measure of accuracy, it is therefore expected to calculate the forecast error, in percentage values.

According to what has been attained in the results for the ANN, in the different scenarios, is possible to underline that the results were quite promising. Both images predicted the pumping power in the periods that were expected, although having an error associated between the Actual Pump and Pumping Process. The results differ from the targets, due to the selection of the input data or the few scenarios that were considered, which could lead to a little consistency in the learning process. 
These results were important to comprehend how an ANN works and how is developed. As said before, the selection of the input parameters are vital and represent a crucial part in the development of a solution. The higher is the detail in the data processing, higher accuracy and convergence time it will be from the output.

\section{CONCLUSION}

One of the problems that has becoming more and more interesting to approach in fields of study is the constant increase of wind penetration in the electric network. The main objective undergoes by preserving the growth of renewable energy in the society and putting aside, each time more, the non-renewable energy. Another aspect that is important to take into account is the increase of distributed generation and Demand Response programs, which has leading to new challenges for electricity markets operators. To complement, is extremely necessary to redefine the way the electricity network is managed and operated, in order to preserve its stability and efficiency.

It is vital to point out that the proposed methodology and the obtained results were crucial to comprehend the impressive advantages of these methods when applied to a real scenario, in this case to the Portuguese Power System. The programs of Demand Response and Real-Time Pricing proved to be efficient in the terms in which they were used.

The VPP proved to be competent in the minimization of energy costs, regarding the resources scheduling, through the constant measure of the demand state. One of the most important aspects of the VPP is also to bring stability to the electric network.

The artificial neural network, in this context, it is very important, because it is a tool that can be easily applied, whenever the reservoir operator decide in which period it is more viable to turbine the water stored in the reservoir. In this way the ANN performs a quick prediction to know how much power should be stored, in the reservoir, and in which periods. To conclude, the use of ANN proved to be interesting for solving daily problems and was also possible to generate good results.

\section{REFERENCES}

[1] Venayagamoorthy, G.K., "Dynamic, Stochastic, Computational, and Scalable Technologies for Smart Grids," Computational Intelligence Magazine, IEEE , vol.6, no.3, pp.22,35, Aug. 2011

[2] EWEA, "Wind in power - 2012 European statistics", February, 2013, available online:

http://www.ewea.org/fileadmin/files/library/statistics/Wind_in_power_a nnual_statistics_2012.pdf

[3] EWEA, "Wind in power - 2013 European statistics", February, 2014, available online: http://www.ewea.org/fileadmin/files/library/publications/statistics/EWE A_Annual_Statistics_2013.pdf

[4] EWEA, "Wind in power - 2014 European statistics", February, 2015, available online:

http://www.ewea.org/fileadmin/files/library/publications/statistics/EWE A-Annual-Statistics-2014.pdf

[5] Boldt, Diogo; Faria, Pedro; Vale, Zita, "Study and analysis of wind curtailment situations and developing an appropriated methodology for its management," in PowerTech, 2015 IEEE Eindhoven, vol., no., pp.1-6, June 29 2015-July 22015

[6] Pedro Faria, Zita Vale, Jose Baptista, "Constrained consumption shifting management in the distributed energy resources scheduling considering demand response", Energy Conversion and Management, Volume 93, 15 March 2015, Pages 309-320, ISSN 0196-8904

[7] P. Brown, J. Lopes and M. Matos, "Optimization of pumped storage capacity in an isolated power system with large renewable penetration", IEEE Transactions on Power Systems, vol. 23, pp. 523-531, May 2008.

[8] Aidan Tuohy and Mark O'Malley, "Impact of pumped storage on power systems with increasing wind penetration", in Proc. IEEE Power and Energy Society General Meeting, vol. 1, pp. 1-6, Jul. 2009

[9] "Neural Network Primer: Part I" by Maureen Caudill, AI Expert, Feb. 1989

[10] McCarthy's definition of AI: McCarthy 2007

[11] S. Haykin, "Neuronal Networks, A Comprehensive Fundation, Second Edition", McMaster University, Hamilton, Ontario, Canada, 1999, Prentice Hall International, Inc.

[12] Jain, A.K.; Jianchang Mao; Mohiuddin, K.M., "Artificial neural networks: a tutorial," Computer, vol.29, no.3, pp.31,44, Mar 1996

[13] "TOMLAB User Guide", Matlab, available online: http://tomopt.com/docs/TOMLAB.pdf

[14] H. Morais, T. Pinto, Z. Vale, I. Praça, Multilevel Negotiation in Smart Grids for VPP Management of Distributed Resources, IEEE Intelligent Systems, vol. 27, no. 6, pp. 8-16, 2012.

[15] "Neural Network Toolbox ${ }^{\mathrm{TM}}$ User's Guide", Matlab https://www.mathworks.com/help/pdf.doc/nnet/nnet_ug.pdf

[16] REN, "Estatístisca diária", available online: http://www.centrodeinformacao.ren.pt/PT/InformacaoExploracao/Pagina s/EstatisticaDiariaDiagrama.aspx, visited on 9th March 2014, visited on 9th March 2014. 